\title{
The hematopoietic stem cell diet
}

\author{
Adam C. Wilkinson ${ }^{1,2} \cdot$ Satoshi Yamazaki ${ }^{3}$
}

Received: 10 March 2018 / Accepted: 27 March 2018 / Published online: 31 March 2018

(c) The Japanese Society of Hematology 2018

\begin{abstract}
Hematopoietic stem cells (HSCs) are responsible for sustaining life-long blood formation or hematopoiesis and are also used clinically in a form of bone marrow transplantation, a curative cellular therapy for a range of hematological diseases. HSCs are maintained throughout adult life by a complex biological niche or microenvironment, which is thought to be composed of a range of cellular, molecular, and metabolic components. The metabolic components of the HSC niche have become of increasing interest over the past few years. It is now well-recognized that metabolic activity is intimately linked to HSC function, and dysregulation of these metabolic pathways result in hematological pathologies such as leukemia. Here, we review the recent progress in this field including our current understanding of the "dietary" requirements of HSCs and how nutrition influences HSC activity. These recent findings have suggested promising new metabolic approaches to improve clinical HSC transplantation and leukemia therapies.
\end{abstract}

Keywords Hematopoietic stem cell $\cdot$ Metabolism $\cdot$ Nutrition $\cdot$ HSC $\cdot$ Hematopoietic stem cell transplantation $\cdot$ Leukemia

\section{Introduction}

The blood or hematopoietic system plays numerous essential roles in human health and disease. Failure in blood system homeostasis results in a range of human diseases, from anemia and hemophilia, to immunodeficiency and allergy, or leukemia and lymphoma. Blood system homeostasis is sustained by hematopoietic stem and progenitor cells (HSPCs), which are mainly found within a complex bone marrow (BM) microenvironment or niche. Hematopoietic stem cells (HSCs) are thought to exist at the top of the hematopoietic "lineage tree" due to their multipotent differentiation capacity (allowing them to generate any adult blood lineage), and self-renewal capacity (the ability to replicate

Satoshi Yamazaki

y-sato4@ims.u-tokyo.ac.jp

1 Institute for Stem Cell Biology and Regenerative Medicine, Stanford University School of Medicine, Lorry I. Lokey Stem Cell Research Building, 265 Campus Drive, Stanford, CA, USA

2 Department of Genetics, Stanford University, Stanford, CA, USA

3 Division of Stem Cell Therapy, Institute of Medical Science, University of Tokyo, Tokyo 108-8639, Japan themselves) [1, 2]. However, HSCs are rare and largely quiescent during homeostasis [3, 4]. Steady-state hematopoiesis is instead thought to be sustained by hematopoietic progenitors (HPCs), the progeny of HSCs [5], which proliferate and differentiate into mature blood cells through progressively more lineage-restricted cell states [6]. The maintenance and differentiation of HSPCs is tightly regulated by both intrinsic $[7,8]$ and extrinsic $[9,10]$ mechanisms. Dysregulation of these processes is thought to lead to the age-related decline in blood system function and related HSC aging, as well as hematological disease.

HSCs have been the focus of considerable clinical and biomedical research [11]. The unique multipotency and selfrenewal capacities of HSCs mean that following transplantation into a recipient, HSCs can reconstitute the entire blood system and sustain hematopoiesis long-term. This is basis of clinical BM or HSC transplantation (HSCT), which is a curative therapy for a range of hematological diseases [12] (including those mentioned above). Donor HSC engraftment and blood system reconstitution is dependent there being "space" in the recipient's HSC BM niche. While a number of BM conditioning regimens have now been suggested, in the clinical setting, it is usually achieved by chemotherapy and/or irradiation [11]. With the aim of improving HSCT and understanding mechanisms underlying hematological 
diseases, much effort has been spent identifying physical constituents of the HSC niche. It is worth noting that HSPCs have been identified in other tissues such as the spleen, muscle, liver, and more recently lung $[13,14]$. The HSC niche provides the necessary molecular and metabolic interactions that sustain HSC function. Various cell types have now been identified as HSC niche components, which have been reviewed extensively elsewhere $[9,10]$.

It has long been known that dietary and metabolic changes alter hematopoiesis. For example, Arthur Kornberg and others demonstrated over 60 years ago that amino acids were required for granulocyte and erythrocyte production [15]. However, only more recently has the metabolic requirements for HSC function become a focus of research. Here, we review the recent literature connecting HSCs and our diets, with a focus the last 2 years. This includes an overview of our understanding of the metabolic requirement of HSCs, a summary of how nutrient-sensing pathways modulate HSC function, and a discussion of recently proposed nutritional approaches to improve HSCT and treat leukemia.

\section{The metabolic activity of HSCs}

While adult HSCs are largely dormant (quiescent) under steady-state conditions, HSCs become activated and enter the cell cycle following injury, stress, or transplantation, to re-establish blood system homeostasis [16]. As discussed below, dormant and cycling HSCs have different metabolic activities and nutritional demands (Fig. 1).

\section{Nutritional requirements of dormant and cycling HSCS}

Dormant HSCs are thought to have low energy and biosynthetic requirements, as compared to actively dividing cells $[17,18]$. Additionally, the HSC niche within the BM is hypoxic, and is thought to limit oxidative phosphorylation (aerobic respiration). Hypoxia and associated hypoxiainducible factor (HIF) activity are thought to play an important role in maintenance of HSC self-renewal [18-20]. As reviewed elsewhere $[17,18]$, HSCs are therefore thought to heavily rely on anaerobic respiration, and genetic inhibition of glycolysis has been shown to inhibit HSC maintenance [21]. By contrast, proliferation is linked to oxidative phosphorylation [17]. While HSCs appear to require glycolysis to maintain function, Ito et al. have suggested that fatty oxidation also plays an important role in HSC maintenance [22]. Specifically, fatty acid oxidation was shown to regulate HSC fate decisions during cell division, implicating lipid metabolism in self-renewal.

HSCs have also recently been found to exhibit low rates of protein translation, suggesting a lower requirement for

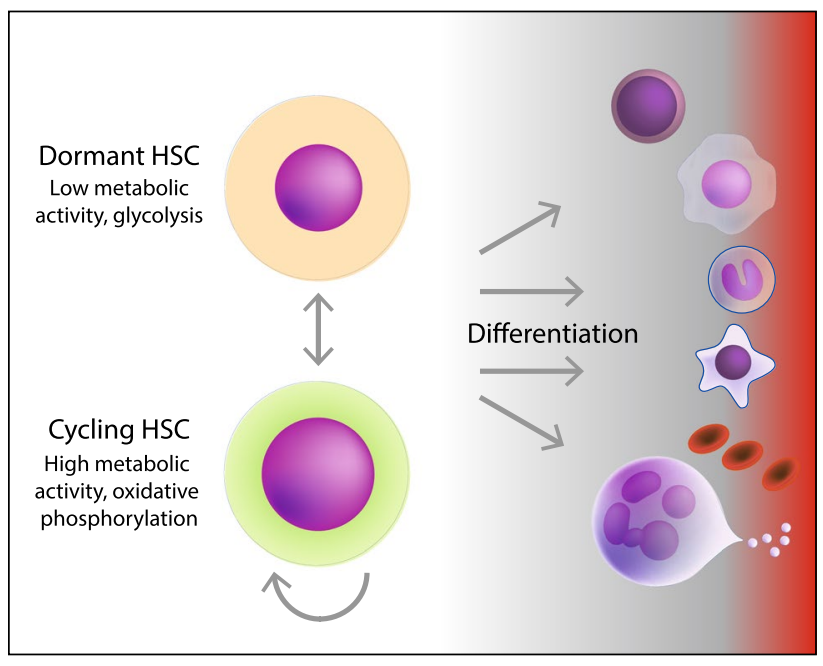

Fig. 1 Cellular and metabolic activity of hematopoietic stem cells. Hematopoietic stem cells (HSCs) are largely dormant under steadystate conditions but can become activated and enter the cell cycle in response to stress, injury, or transplantation. HSCs can self-renew or differentiate into the various mature blood lineages to sustain blood production throughout life. Dormant and cycling HSCs have distinct metabolic activities, and nutritional changes can influence these HSC fate decisions

amino acids than other proliferative cell types for protein synthesis [23]. However, in a recent study, Taya et al. found that in vivo HSC maintenance was dependent on the amino acid valine [24]. Ex vivo HSC proliferation was also dependent on valine as well as cysteine. By contrast, reducing threonine improved ex vivo HSC maintenance, suggesting threonine (or downstream metabolites) could be playing a role negative role in HSC function. While cysteine was found to play an important role in biosynthesis of the antioxidant glutathione [24], the metabolic roles for valine and threonine remain an open question. In a separate recent study, glutamine was shown to regulate HSC commitment into erythroid differentiation [25], where it appears to play an important role in de novo nucleotide biosynthesis.

Vitamins are essential nutrients necessary for human health. Roles for vitamins A, C, and D in HSC maintenance have been recently been identified [26-29]. Cabezas-Wallscheid et al. recently reported that vitamin A (and its oxidation-derivative retinol) regulated HSC dormancy. In mice, a vitamin A-free diet caused loss of HSCs following stress activation, by inhibiting the re-entry of HSCs into dormancy [26]. Vitamin D was recently shown to regulate HSC proliferation and self-renewal, as well as developmental HSC formation in zebrafish [27]. By contrast, depletion of vitamin $\mathrm{C}$ in mice was shown to increase HSC frequency and function [29]. Vitamin C is a co-factor for the enzymatic activity of the TET family of DNA hydroxylases, of which TET2 is a well-described negative regulator of HSC self-renewal [30]. Vitamin C is thought to act via TET2-dependent and 
independent pathways to limit self-renewal [29]. As discussed below, vitamin $\mathrm{C}$ was also found to regulate leukemogenesis [29, 31].

\section{Mitochondrial activity and autophagy regulate HSC function}

Consistent with the idea that HSCs were heavily reliant on glycolysis, transplantable HSCs were found to have low mitochondrial activity [20]. However, while HSCs have low mitochondrial activity, they appear to have high mitochondrial mass [32]. Interestingly, genetic deletion of the RISP subunit of the mitochondrial electron transport chain complex III has recently been shown to cause loss of adult HSC quiescence and function, resulting in pancytopenia and lethality [33]. The importance of mitochondrial fusion for HSC lymphoid potential has also recently been identified by genetic deletion of Mitofusin2 [34]. These recent findings highlight the importance of mitochondrial activity in HSC function.

While HSCs can receive nutrients from their extracellular environment, cells can also recycle cytoplasmic proteins and organelles through autophagy, and even mitochondria by the related process of mitophagy. Interestingly, although HSCs reside in what is thought of as a nutrient-rich BM microenvironment [24], autophagy and mitophagy are both implicated in HSC maintenance [35-37]. Autophagy has recently been shown to play an important role in HSC quiescence, with loss of autophagy resulting in an activated metabolic state, which inhibited self-renewal and promoted differentiation. HSC self-renewal is also thought to rely on mitophagy [37]. Additionally, aged HSCs were found to display reduced autophagy and this has been proposed to play a mechanistic role in the age-related decline in HSC function [35]. Therefore, although HSCs heavily rely on the BM microenvironment for nutrients; it is now clear that metabolic recycling also plays an important role in HSC maintenance.

\section{Nutrient-sensing pathways regulate HSC function}

Several studies have now linked dietary nutrition to HSC activity, highlighting the importance of nutrition on blood system homeostasis. However, as discussed below, these studies have largely focused on the role of nutrient-sensing pathways and the changing composition of HSC niche cells within the BM in these contexts.

\section{Fasting and diabetes}

One of the first demonstrations of the power of nutrition to enhance HSC function was provided by Cheng et al. who demonstrated that prolonged fasting (cycles of 48-h fasting) promoted HSC regeneration in mice [38]. Cheng et al. found that prolonged fasting acted via inhibition of insulin growth factor 1 (IGF1) signaling. It is worth noting that the kinetics of fasting likely play an important role in this regenerative phenotype because it was recently reported that chronic (life-long) calorific restriction does not alter HSC function [39]. A fasting-like state is also seen in diabetes. Interestingly, a retrospective analysis of patient records by Ferraro et al. found that diabetes negatively correlated with donor HSPC mobilization for HSCT [40]. Ferraro et al. went on to demonstrate that HSCs poorly mobilized in mouse models of diabetes, resulting in higher HSC frequencies in the BM. It was suggested that diabetes regulated HSC mobilization via the CXCL12-CXCR4 axis, a key HSC niche interaction [14].

\section{High-fat diets and obesity}

While fasting appears to enhance HSC function, a number of recent reports have found that high-fat diets and obesity reduce HSC activity [41-43]. For example, diet-induced obesity is thought to promote HSC differentiation, particularly myeloid differentiation, at the expense of self-renewal [41, 42]. This leads to poor hematopoietic stress recovery, such as following treatment of the chemotherapy agent 5-fluorouracil [43]. It is thought that adipocytes (fat storage cells) play an important role in mediating this interaction [44]. Adipocytes are found within the BM, and adipocyte-HSC interactions have been recently identified [45]. For example, adipocytes have been found to transiently expand following BM injury such as irradiation, where they provide cytokines such as stem cell factor (SCF) to promote hematopoietic system regeneration [45]. However, accumulation of adipocytes within the BM in obesity or during aging is thought to impair HSC function [44]. Acute myeloid leukemia (AML) has also recently been suggested to drive bone marrow failure by perturbing BM adipocyte function [46].

\section{HSC-microbiome interactions}

The microbiome is the symbiotic (and sometimes pathogenic) community of microorganisms in our bodies that are now known to play important roles in regulating mammalian health and disease [47]. The microbiome performs a number of important metabolic and immunological functions, particularly those residing within the gut [47]. Recently, novel microbiome-HSC axes have been identified [48, 49]. For example, the microbiome associated with obesity plays a role in promoting the BM adipocyte changes described above, which promote HSC differentiation [48]. The microbiome is also thought to influence HSC activity via BM 
mesenchymal stromal cells, another well-described HSC niche component [49]. Microbiome-hematopoietic system interactions are also thought to underlie the BM suppression induced by chronic antibiotic treatment, which causes microbiome depletion and results in loss of HSPCs, anemia, and pancytopenia [50]. Given the importance of the hematopoietic system in immune function, these studies highlight the importance of a healthy microbiome and the potentially detrimental consequences of antibiotic intervention.

\section{HSC nutrient stress sensing pathways}

Nutrient availability regulates the activity of nutrient stress sensing pathways, which in turn promotes or limits metabolic activity including protein synthesis [51]. These include the mammalian target of rapamycin (mTOR) [52] and integrated stress response (ISR) pathways [53]. Nutrient availability directly (and indirectly) regulate the activity of mTOR complex 1 (mTORC1), a central node in metabolic sensing and signaling [51]. A number of studies have demonstrated the importance of mTOR in HSC function, and have been recently reviewed elsewhere $[54,55]$. Availability of several nutrients, including the essential amino acid leucine, is thought regulate mTORC1 activity via the GTPase RagA [52]. Interestingly, RagA was recently found to be dispensable for HSC function [56], suggesting HSCs to be resilient to certain nutrient stresses.

The ISR pathway integrates a number of cellular stresses including amino acid availability and endoplasmic reticulum stress via phosphorylation of translational initiation factor 2a (eIF2a) to regulate protein synthesis, stress response genes, and ultimately apoptosis [53]. Interestingly, HSCs are thought to have high ISR activity under steady-state conditions, which makes them sensitive to additional ISR stresses [57]. It has been suggested that this ISR sensitivity helps to maintain integrity of the HSC pool by preventing expansion of damaged HSCs [57]. Therefore, while nutrients are substrates for cellular biosynthesis, it is also worth remembering that nutrients also act as signaling molecules that influence transcriptional and translational programs.

\section{Metabolic opportunities to improve HSCT}

While HSCT represents a potentially curative therapy for a range of hematological diseases, several challenges remain that can limit the use of this approach. These include the limited availability of donor HSCs for stable engraftment and the toxicity associated with current chemotherapy/irradiation-based BM conditioning. Over the last few years, a several metabolic approaches have been identified that may help to overcome these clinical challenges.

\section{Metabolic approaches to HSC mobilization and expansion}

Collecting sufficient numbers of HSCs from healthy donors without risk remains a major challenge for HSCT. HSCs can be collected by BM aspiration but more often are collected from the peripheral blood following HSC mobilization [58]. Traditionally, HSCs have been mobilized using granulocyte-colony stimulating factor (G-CSF) [58]. However, Oguro et al. recently found that cholesterol derivative, 27-hydroxycholesterol-induced HSC mobilization, and was synergistic with G-CSF, suggesting a novel way to improve HSC mobilization [59]. As relatively few HSCs can be harvested from a healthy donor, expanding HSCs ex vivo represents a rational strategy to improve transplantation dose [60]. Unfortunately, we are still unable to stably expand HSCs ex vivo, with HSCs quickly losing potency [60]. Interestingly, Guo et al. recently reported that enhancing glycolysis substantially improved human HSC expansion ex vivo [61]. These data suggest that through our research into HSC metabolism and nutrient dependencies, we may be able to further optimize conditions that sustain and expand HSCs for HSCT.

\section{Metabolic approaches to BM conditioning}

BM conditioning is a necessary step in HSCT to make space for donor HSCs to engraft [12]. The recent finding that HSCs were dependent on valine, has led to the proposal of metabolic BM conditioning approaches [24]. For example, dietary depletion of valine in mice for just 2-3 weeks resulted in loss of host HSCs and afforded donor HSC engraftment. Importantly, following treatment, all recipients recovered to full health and fertility, an outcome never achieved following irradiation conditioning [24]. While optimization of such a metabolic BM conditioning approach is required, these findings highlight the promise of safe metabolic and nutritional approaches in HSCT [62].

\section{Metabolic opportunities to improve leukemia treatments}

Leukemia research is a broad and mature research field with leukemia encompasses a large number of genetically and phenotypically distinct blood cancers. Here, we focus on recently reported approaches to treat leukemias through metabolic approaches, with an emphasis on those that target nutritional dependencies of malignant HSCs, or leukemic stem cells (LSCs). LSCs are thought to retain many of the properties of HSCs (e.g., self-renewal, quiescence), 
which allow them to resist chemotherapy treatments and drive disease relapse in patients [63].

\section{Metabolic regulation of TET2 activity in leukemia}

The identification of genetic mutations in the metabolic enzymes isocitrate dehydrogenase 1 (IDHI) and 2 (IDH2) in AML [64] led to a search for mechanisms of metabolic corruption in leukemogenesis [65]. Wild-type IDH enzymes catalyze production of alpha-ketoglutarate, which plays a range of biological functions including as a substrate for TET2. By contrast, mutant IDH enzymes were found to catalyze production of 2-hydroxyglutarate, which acts as an inhibitor of TET2 activity [66]. Genetic mutation or loss-of-function of TET2 plays a well-described role in aberrant self-renewal and leukemogenesis [30], and is one of the most commonly mutated genes in AML [64]. As mentioned above, vitamin $\mathrm{C}$ also regulates TET2 enzymatic activity and its depletion enhanced self-renewal activity [29]. Vitamin C depletion also accelerated leukemogenesis while high vitamin $\mathrm{C}$ inhibited leukemic progression [29, 31]. These findings highlight how nutritional status can regulate leukemogenesis through influencing epigenetic regulators of self-renewal.

\section{Dysregulation of branched-chain amino acid metabolism in leukemia}

Leucine, isoleucine, and valine are all branched-chain amino acids (BCAAs), a subset of essential amino acids. Besides their role in protein synthesis, BCAAs are thought to play a number of other important biological functions, such as in cellular nitrogen balance and energy production, and as mentioned above, the regulation of mTOR activity [67]. The enzymes responsible for the early steps of BCAA catabolism are shared, and include branched-chain aminotransferase 1 (BCAT1), which converts BCAAs into branched-chain ketoacids by transferring the BCAA amino group onto alpha-ketoglutarate (generating glutamate). It has been recently demonstrated that $B C A T 1$ is overexpressed in a subset of AMLs and chronic myeloid leukemias (CMLs) [68, 69]. BCAT1 is overexpressed in TET2/IDH wild-type AML LSCs, where was shown to deplete its substrates BCAAs and alpha-ketoglutarate (Fig. 2) [68]. Here, it has been suggested to drive LSC self-renewal and leukemogenesis at least in part by reducing TET 2 activity (as mentioned above, alphaketoglutarate is also a substrate for TET2).

In CML, an alternative mechanism for $B C A T 1$ overexpression has been proposed [69]. BCAT1 catalyzes a reversible reaction, and can therefore be driven in reverse, generating BCAAs from branched-chain ketoacids (Fig. 2). This

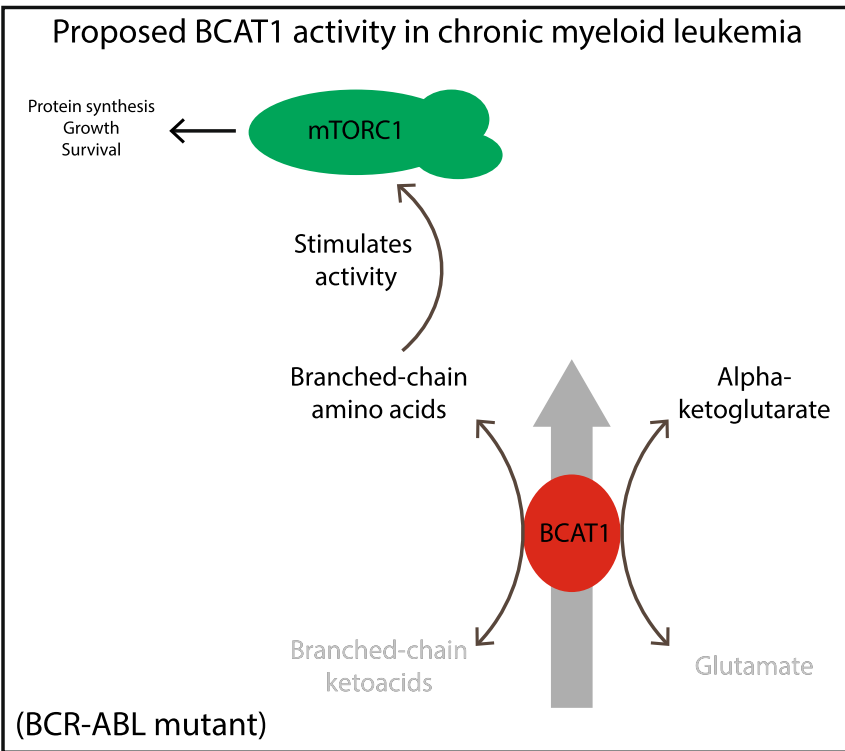

Proposed BCAT1 activity in acute myeloid leukemia

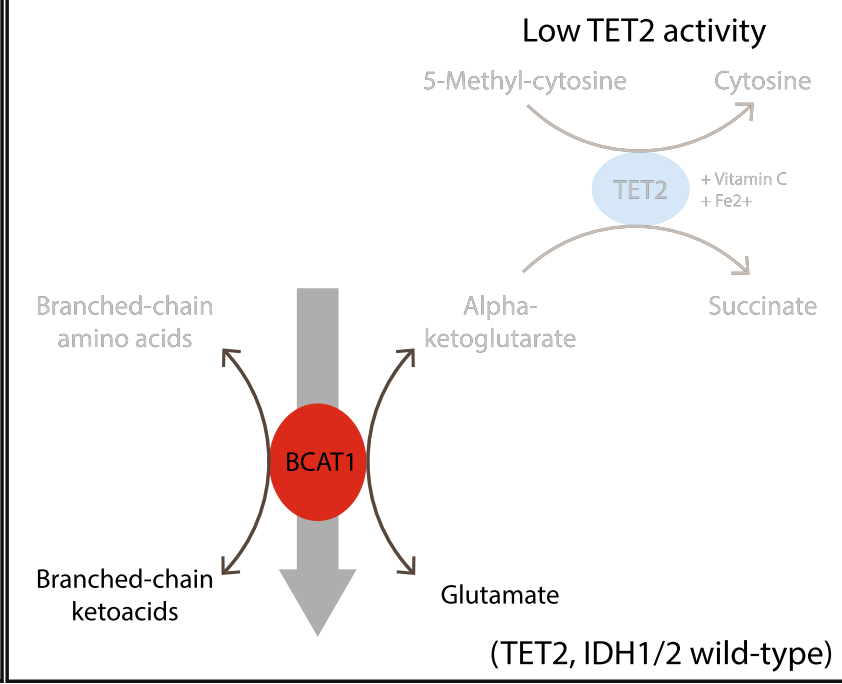

Fig. 2 Emerging insights into branched-chain amino acid metabolism in leukemia. Chronic myeloid leukemia (CML) and acute myeloid leukemia (AML) leukemia stem cells overexpress the first enzyme in branched-chain amino acid (BCAA) metabolism, branched-chain aminotransferase 1 (BCAT1), which appears to play context-dependent functions in leukemogenesis. In CML (left panel), BCAT1 has been suggested to increase cellular concentrations of BCAAs to stimulate mTOR activity, to drive protein synthesis, growth, and survival.

In AML with genetically wild-type TET2 and IDH1/2 (right panel), BCAT1 has been proposed to catabolize BCAAs and alpha-ketoglutarate. The resulting reductions in alpha-ketoglutarate are thought to limit the activity of the epigenetic regulator TET2 (alpha-ketoglutarate is a substrate for its enzymatic activity), a negative regulator of self-renewal, thereby mimicking genetic TET2 loss-of-function mutations that are common in AML 
was proposed by Hattori et al. who found that in BCR-ABL driven CML, BCAT1 overexpression led to increased intracellular BCAA concentrations [69]. The BCAA leucine is a well-described mTORC1 agonist and the corresponding mTORC1 activity was demonstrated, which was suggested to promote aberrant proliferation and survival. Importantly, in both AML and CML displaying high BCATl expression, inhibition of BCAT1 activity blocked leukemogenesis and disease progression $[68,69]$. These studies suggest BCAT1 and BCAA metabolism as a therapeutic target in leukemia.

\section{Approaches to starving leukemia}

As mentioned above, fasting has been shown to enhance HSC function [38]. Interestingly, fasting has also recently been found to block the development of acute lymphoblastic leukemia (ALL) [70]. It was further demonstrated that fasting prevented ALL development via blocking the upregulation of the leptin receptor. However, it is worth noting that fasting did not inhibit development of AML, suggesting disease-specific dependencies. By contrast, glutamine starvation has recently been suggested to synergize with tyrosine kinase inhibitors to inhibit certain myeloid leukemias [71]. Other researchers have also attempted to pharmacologically "starve" leukemias, such as using inhibitors of fatty acid oxidation. For example, pharmacological inhibition of carnitine palmitoyl transferase 1 , a rate limiting enzyme in fatty acid oxidation, has proved effective in blocking leukemic proliferation [72]. Starvation can also be mimicked using mTOR inhibitions, which have also shown promise in inhibiting several leukemias [55]. Better understanding the different metabolic requirements of HSCs and LSCs will help to identify new targetable metabolic vulnerabilities for the safe treatment and cure of leukemia.

\section{Conclusions}

While the importance of good nutrition for a healthy blood system is well-recognized, we have only recently started to appreciate the complex metabolic interplay that regulates HSC activity and function. So far, a number of exciting metabolic approaches have already been proposed to help improve HSCT and treat leukemias. Most research todate has focused on metabolism of adult BM HSCs. Much remains to be learnt about the metabolic activity at other HSC sites [14]. Additionally, we are only just starting to understand the metabolic requirements for embryonic HSC development [27], but such findings may help efforts to derive HSCs from pluripotent stem cells ex vivo. In other systems, metabolic activity has been recently shown to differ widely between the developmental stages [73], suggesting embryonic and adult HSCs may also display distinct metabolic dependencies. We have also only recently begun to understand the role of metabolism in HSC aging [35, 44]. Given the fast-pace of HSC metabolism research today, we are confident that new metabolic approaches to modulate healthy and malignant HSCs will soon be identified, and that in the longer term these research efforts will improve the lives of patients with hematological diseases.

Acknowledgements We thank H. J. Beker and other members of the Yamazaki laboratory for feedback on the manuscript. We apologize to those authors whose work could not be cited due to space constraints. SY is supported by the Japan Society for the Promotion of Science (JSPS) (Grant no. 50625580), and the Ministry of Education, Culture, Sport, Science, and Technology (Japan). ACW is supported by Bloodwise (15050) and the NIH National Center for Advancing Translational Science Clinical and Translational Science Award (UL1 TR001085). The content is solely the responsibility of the authors and does not necessarily represent the official views of the NIH.

\section{References}

1. Osawa M, Hanada K, Hamada H, Nakauchi H. Long-term lymphohematopoietic reconstitution by a single CD34-low/negative hematopoietic stem cell. Science. 1996;273(5272):242-5.

2. Laurenti E, Göttgens B. From haematopoietic stem cells to complex differentiation landscapes. Nature. 2018;553(7689):418-26.

3. Cheshier SH, Morrison SJ, Liao X, Weissman IL. In vivo proliferation and cell cycle kinetics of long-term self-renewing hematopoietic stem cells. Proc Natl Acad Sci USA. 1999;96(6):3120-5.

4. Sudo K, Ema H, Morita Y, Nakauchi H. Age-associated characteristics of murine hematopoietic stem cells. J Exp Med. 2000;192(9):1273-80.

5. Sun J, Ramos A, Chapman B, et al. Clonal dynamics of native haematopoiesis. Nature. 2014;514(7522):322-7.

6. Yamamoto R, Morita Y, Ooehara J, et al. Clonal analysis unveils self-renewing lineage-restricted progenitors generated directly from hematopoietic stem cells. Cell. 2013;154(5):1112-26.

7. Wilkinson AC, Gottgens B. Transcriptional regulation of haematopoietic stem cells. Adv Exp Med Biol. 2013;786:187-212.

8. Wilkinson AC, Nakauchi H, Göttgens B. Mammalian transcription factor networks: recent advances in interrogating biological complexity. Cell Syst. 2017;5(4):319-31.

9. Morrison SJ, Scadden DT. The bone marrow niche for haematopoietic stem cells. Nature. 2014;505(7483):327-34.

10. Boulais PE, Frenette PS. Making sense of hematopoietic stem cell niches. Blood. 2015;125(17):2621-9.

11. Eaves CJ. Hematopoietic stem cells: concepts, definitions, and the new reality. Blood. 2015;125(17):2605-13.

12. Copelan EA. Hematopoietic stem-cell transplantation. N Engl J Med. 2006;354(17):1813-26.

13. Lefrançais E, Ortiz-Muñoz G, Caudrillier A, et al. The lung is a site of platelet biogenesis and a reservoir for haematopoietic progenitors. Nature. 2017;544(7648):105-9.

14. Crane GM, Jeffery E, Morrison SJ. Adult haematopoietic stem cell niches. Nat Rev Immunol. 2017;17(9):573-90.

15. Kornberg A. Amino acids in the production of granulocytes in rats. J Biol Chem. 1946;164:203-12.

16. Pietras EM, Warr MR, Passegué E. Cell cycle regulation in hematopoietic stem cells. J Cell Biol. 2011;195(5):709-20.

17. Karigane D, Takubo K. Metabolic regulation of hematopoietic and leukemic stem/progenitor cells under homeostatic and stress conditions. Int J Hematol. 2017;106(1):18-26. 
18. Suda T, Takubo K, Semenza GL. Metabolic regulation of hematopoietic stem cells in the hypoxic niche. Cell Stem Cell. 2011;9(4):298-310.

19. Takubo K, Goda N, Yamada W, et al. Regulation of the HIF1 alpha level is essential for hematopoietic stem cells. Cell Stem Cell. 2010;7(3):391-402.

20. Simsek T, Kocabas F, Zheng J, et al. The distinct metabolic profile of hematopoietic stem cells reflects their location in a hypoxic niche. Cell Stem Cell. 2010;7(3):380-90.

21. Takubo K, Nagamatsu G, Kobayashi CI, et al. Regulation of glycolysis by Pdk functions as a metabolic checkpoint for cell cycle quiescence in hematopoietic stem cells. Cell Stem Cell. 2013;12(1):49-61.

22. Ito K, Carracedo A, Weiss D, et al. A PML-PPAR- $\delta$ pathway for fatty acid oxidation regulates hematopoietic stem cell maintenance. Nat Med. 2012;18(9):1350-8.

23. Signer RA, Magee JA, Salic A, Morrison SJ. Haematopoietic stem cells require a highly regulated protein synthesis rate. Nature. 2014;509(7498):49-54.

24. Taya Y, Ota Y, Wilkinson AC, et al. Depleting dietary valine permits nonmyeloablative mouse hematopoietic stem cell transplantation. Science. 2016;354(6316):1152-5.

25. Oburoglu L, Tardito S, Fritz V, et al. Glucose and glutamine metabolism regulate human hematopoietic stem cell lineage specification. Cell Stem Cell. 2014;15(2):169-84.

26. Cabezas-Wallscheid N, Buettner F, Sommerkamp P, et al. Vitamin A-retinoic acid signaling regulates hematopoietic stem cell dormancy. Cell. 2017;169(5):807-823.e819.

27. Cortes M, Chen MJ, Stachura DL, et al. Developmental vitamin D availability impacts hematopoietic stem cell production. Cell Rep. 2016;17(2):458-68.

28. Studzinski GP, Harrison JS, Wang X, Sarkar S, Kalia V, Danilenko M. Vitamin D control of hematopoietic cell differentiation and leukemia. J Cell Biochem. 2015;116(8):1500-12.

29. Agathocleous M, Meacham CE, Burgess RJ, et al. Ascorbate regulates haematopoietic stem cell function and leukaemogenesis. Nature. 2017;549(7673):476-81.

30. Moran-Crusio K, Reavie L, Shih A, et al. Tet2 loss leads to increased hematopoietic stem cell self-renewal and myeloid transformation. Cancer Cell. 2011;20(1):11-24.

31. Cimmino L, Dolgalev I, Wang Y, et al. Restoration of TET2 function blocks aberrant self-renewal and leukemia progression. Cell. 2017;170(6):1079-1095.e1020.

32. de Almeida MJ, Luchsinger LL, Corrigan DJ, Williams LJ, Snoeck HW. Dye-independent methods reveal elevated mitochondrial mass in hematopoietic stem cells. Cell Stem Cell. 2017;21(6):725-729.e724.

33. Ansó E, Weinberg SE, Diebold LP, et al. The mitochondrial respiratory chain is essential for haematopoietic stem cell function. Nat Cell Biol. 2017;19(6):614-25.

34. Luchsinger LL, de Almeida MJ, Corrigan DJ, Mumau M, Snoeck HW. Mitofusin 2 maintains haematopoietic stem cells with extensive lymphoid potential. Nature. 2016;529(7587):528-31.

35. Ho TT, Warr MR, Adelman ER, et al. Autophagy maintains the metabolism and function of young and old stem cells. Nature. 2017;543(7644):205-10.

36. Cao Y, Zhang A, Cai J, et al. Autophagy regulates the cell cycle of murine HSPCs in a nutrient-dependent manner. Exp Hematol. 2015;43(3):229-42.

37. Ito $\mathrm{K}$, Turcotte $\mathrm{R}$, Cui $\mathrm{J}$, et al. Self-renewal of a purified $\mathrm{Tie} 2^{+}$hematopoietic stem cell population relies on mitochondrial clearance. Science. 2016;354(6316):1156-60.

38. Cheng CW, Adams GB, Perin L, et al. Prolonged fasting reduces IGF-1/PKA to promote hematopoietic-stem-cell-based regeneration and reverse immunosuppression. Cell Stem Cell. 2014;14(6):810-23.
39. Lazare S, Ausema A, Reijne AC, van Dijk G, van Os R, de Haan G. Lifelong dietary intervention does not affect hematopoietic stem cell function. Exp Hematol. 2017;53:26-30.

40. Ferraro F, Lymperi S, Méndez-Ferrer S, et al. Diabetes impairs hematopoietic stem cell mobilization by altering niche function. Sci Transl Med. 2011;3(104):104ra101.

41. Singer K, DelProposto J, Morris DL, et al. Diet-induced obesity promotes myelopoiesis in hematopoietic stem cells. Mol Metab. 2014;3(6):664-75.

42. van den Berg SM, Seijkens TT, Kusters PJ, et al. Diet-induced obesity in mice diminishes hematopoietic stem and progenitor cells in the bone marrow. FASEB J. 2016;30(5):1779-88.

43. Li Y, Zhu S, Zhang Y, et al. High fat diet-induced obesity exacerbates hematopoiesis deficiency and cytopenia caused by 5 -fluorouracil via peroxisome proliferator-activated receptor $\gamma$. Exp Hematol. 2018. https://doi.org/10.1016/j.exphem.2017.12.013.

44. Ambrosi TH, Scialdone A, Graja A, et al. Adipocyte accumulation in the bone marrow during obesity and aging impairs stem cell-based hematopoietic and bone regeneration. Cell Stem Cell. 2017;20(6):771-784.e776.

45. Zhou BO, Yu H, Yue R, et al. Bone marrow adipocytes promote the regeneration of stem cells and haematopoiesis by secreting SCF. Nat Cell Biol. 2017;19(8):891-903.

46. Boyd AL, Reid JC, Salci KR, et al. Acute myeloid leukaemia disrupts endogenous myelo-erythropoiesis by compromising the adipocyte bone marrow niche. Nat Cell Biol. 2017;19(11):1336-47.

47. Lloyd-Price J, Abu-Ali G, Huttenhower C. The healthy human microbiome. Genome Med. 2016;8(1):51.

48. Luo Y, Chen GL, Hannemann N, et al. Microbiota from obese mice regulate hematopoietic stem cell differentiation by altering the bone niche. Cell Metab. 2015;22(5):886-94.

49. Iwamura C, Bouladoux N, Belkaid Y, Sher A, Jankovic D. Sensing of the microbiota by NOD1 in mesenchymal stromal cells regulates murine hematopoiesis. Blood. 2017;129(2):171-6.

50. Josefsdottir KS, Baldridge MT, Kadmon CS, King KY. Antibiotics impair murine hematopoiesis by depleting the intestinal microbiota. Blood. 2017;129(6):729-39.

51. Efeyan A, Comb WC, Sabatini DM. Nutrient-sensing mechanisms and pathways. Nature. 2015;517(7534):302-10.

52. Saxton RA, Sabatini DM. mTOR signaling in growth, metabolism, and disease. Cell. 2017;168(6):960-76.

53. Pakos-Zebrucka K, Koryga I, Mnich K, Ljujic M, Samali A, Gorman AM. The integrated stress response. EMBO Rep. 2016;17(10):1374-95.

54. Meng D, Frank AR, Jewell JL. mTOR signaling in stem and progenitor cells. Development. 2018;145(1):dev152595.

55. Ghosh J, Kapur R. Role of mTORC1-S6K1 signaling pathway in regulation of hematopoietic stem cell and acute myeloid leukemia. Exp Hematol. 2017;50:13-21.

56. Kalaitzidis D, Lee D, Efeyan A, et al. Amino acid-insensitive mTORC1 regulation enables nutritional stress resilience in hematopoietic stem cells. J Clin Investig. 2017;127(4):1405-13.

57. van Galen P, Kreso A, Mbong N, et al. The unfolded protein response governs integrity of the haematopoietic stem-cell pool during stress. Nature. 2014;510(7504):268-72.

58. Domingues MJ, Nilsson SK, Cao B. New agents in HSC mobilization. Int J Hematol. 2017;105(2):141-52.

59. Oguro H, McDonald JG, Zhao Z, Umetani M, Shaul PW, Morrison SJ. 27-Hydroxycholesterol induces hematopoietic stem cell mobilization and extramedullary hematopoiesis during pregnancy. J Clin Investig. 2017;127(9):3392-401.

60. Kumar S, Geiger H. HSC niche biology and HSC expansion ex vivo. Trends Mol Med. 2017;23(9):799-819.

61. Guo B, Huang X, Lee MR, Lee SA, Broxmeyer HE. Antagonism of PPAR- $\gamma$ signaling expands human hematopoietic stem and progenitor cells by enhancing glycolysis. Nat Med. 2018;24:360-7. 
62. Longo VD, Cortellino S. Enhancing stem cell transplantation with "nutri-technology". Cell Stem Cell. 2016;19(6):681-2.

63. Huntly BJ, Gilliland DG. Leukaemia stem cells and the evolution of cancer-stem-cell research. Nat Rev Cancer. 2005;5(4):311-21.

64. Mardis ER, Ding L, Dooling DJ, et al. Recurring mutations found by sequencing an acute myeloid leukemia genome. N Engl J Med. 2009;361(11):1058-66.

65. Dang L, Su SM. Isocitrate dehydrogenase mutation and (R)-2-hydroxyglutarate: from basic discovery to therapeutics development. Annu Rev Biochem. 2017;86:305-31.

66. Xu W, Yang H, Liu Y, et al. Oncometabolite 2-hydroxyglutarate is a competitive inhibitor of $\alpha$-ketoglutarate-dependent dioxygenases. Cancer Cell. 2011;19(1):17-30.

67. Ananieva EA, Wilkinson AC. Branched-chain amino acid metabolism in cancer. Curr Opin Clin Nutr Metab Care. 2018;21(1):64-70.

68. Raffel S, Falcone M, Kneisel N, et al. BCAT1 restricts $\alpha$ KG levels in AML stem cells leading to IDHmut-like DNA hypermethylation. Nature. 2017;551(7680):384-8.
69. Hattori A, Tsunoda M, Konuma T, et al. Cancer progression by reprogrammed BCAA metabolism in myeloid leukaemia. Nature. 2017;545(7655):500-4.

70. Lu Z, Xie J, Wu G, et al. Fasting selectively blocks development of acute lymphoblastic leukemia via leptin-receptor upregulation. Nat Med. 2017;23(1):79-90.

71. Gallipoli P, Giotopoulos G, Tzelepis K, et al. Glutaminolysis is a metabolic dependency in FLT3. Blood. 2018. https://doi. org/10.1182/blood-2017-12-820035.

72. Samudio I, Harmancey R, Fiegl M, et al. Pharmacologic inhibition of fatty acid oxidation sensitizes human leukemia cells to apoptosis induction. J Clin Investig. 2010;120(1):142-56.

73. Koike H, Zhang RR, Ueno Y, et al. Nutritional modulation of mouse and human liver bud growth through a branched-chain amino acid metabolism. Development. 2017;144(6):1018-24. 